\title{
Transarterial and transvenous approaches for embolization of tentorial dural arteriovenous fistula
}

\author{
André Beer-Furlan, MD, Hormuzdiyar H. Dasenbrock, MD, Krishna C. Joshi, MBBS, MCh, \\ and Michael Chen, MD
}

Department of Neurological Surgery, Rush University Medical Center, Chicago, Illinois

Tentorial dural arteriovenous fistulas (DAVFs) are uncommon, complex fistulas located between the leaves of the tentorium cerebelli with a specific anatomic and clinical presentation characterized by high hemorrhagic risk. They have an extensive arterial supply and complex venous drainages, making them difficult to treat. There is recent literature favoring treatment through an endovascular transarterial route. The authors present an uncommon tentorial/ambient cistern region DAVF with feeders arising from the external and internal carotid arteries. The patient underwent a combined transarterial and transvenous approach with successful obliteration of the DAVF. The authors discuss the management challenges faced in this case.

The video can be found here: https://youtu.be/VXDD8zUvsSQ.

KEYWORDS endovascular; dural arteriovenous fistula; tentorial; transarterial; transvenous; ambient cistern; video 